\title{
Auditory steady state cortical responses indicate deviant phonemic-rate processing in adults with dyslexia $^{1}$
}

\author{
Hanne Poelmans $^{\mathrm{a}, \mathrm{b}}$, Heleen Luts ${ }^{\mathrm{a}, \mathrm{b}}$, Maaike Vandermosten ${ }^{\mathrm{a}, \mathrm{b}}$, Bart Boets ${ }^{\mathrm{a}, \mathrm{b}}$, Pol Ghesquière ${ }^{\mathrm{b}}$ \\ and Jan Wouters ${ }^{\mathrm{a}}$
}

${ }^{a}$ ExpORL, Department of Neurosciences, Katholieke Universiteit Leuven, Herestraat 49, 3000 Leuven, Belgium

${ }^{b}$ Parenting and Special Education Research Group, Katholieke Universiteit Leuven, A. Vesaliusstraat 2, 3000 Leuven, Belgium

Corresponding author: Hanne Poelmans, O\&N2, Herestraat 49 bus 721, 3000 Leuven, Belgium. Tel.: +32 163304 95, Fax: +32 163304 86, Hanne.Poelmans@ med.kuleuven.be

\footnotetext{
${ }^{1}$ This article has been published as: Poelmans, H., Luts, H., Vandermosten, M., Boets, B., Ghesquière, P., Wouters, J. (2012). Auditory steady state cortical responses indicate deviant phonemic-rate processing in adults with dyslexia. Ear and Hearing, 33 (1), 134-143.
} 


\begin{abstract}
Objectives: Speech intelligibility is strongly influenced by the ability to process temporal modulations. It is hypothesized that in dyslexia, deficient processing of rapidly-changing auditory information underlies a deficient development of phonological representations, causing reading and spelling problems. Low-frequency modulations between 4 and $20 \mathrm{~Hz}$ correspond to the processing rate of important phonological segments (syllables and phonemes respectively) in speech and therefore provide a bridge between low-level auditory and phonological processing. In the present study, temporal modulation processing was investigated by auditory steady state responses (ASSRs) in normal-reading and dyslexic adults.
\end{abstract}

Design: Multichannel ASSRs were recorded in normal-reading and dyslexic adults in response to speech-weighted noise stimuli amplitude modulated at $80 \mathrm{~Hz}, 20 \mathrm{~Hz}$ and $4 \mathrm{~Hz}$. The $80 \mathrm{~Hz}$ modulation is known to be evoked in the brainstem, whereas the $20 \mathrm{~Hz}$ and $4 \mathrm{~Hz}$ modulations are mainly generated in the cortex. Furthermore, the $20 \mathrm{~Hz}$ and $4 \mathrm{~Hz}$ modulations provide an objective auditory performance measure related to phonemic-rate and syllabic-rate processing. In addition to neurophysiological measures, psychophysical tests of speech-innoise perception and phonological awareness were assessed.

Results: Based on response-strength and phase coherence measures, normal-reading and dyslexic participants showed similar processing at the brainstem level. At the cortical level of the auditory system, dyslexic subjects demonstrated deviant phonemic-rate responses compared to normal readers, whereas no group differences were found for the syllabic-rate. Furthermore, a relation between phonemic-rate ASSRs and psychophysical tests of speech-innoise perception and phonological awareness was obtained.

Conclusions: These results suggest reduced cortical processing for phonemic-rate modulations in dyslexic adults, presumably resulting in limited integration of temporal information in the dorsal phonological pathway. 


\section{Introduction}

Developmental dyslexia refers to a neurological disorder that manifests as a failure to acquire adequate reading and spelling skills despite normal intelligence, education and intense remedial effort and is thought to affect 5 to $10 \%$ of children (Vellutino et al. 2004). Until now, the exact origin of this disorder remains largely unknown. One theory suggests that the reading and writing problems in dyslexia are caused by a deficit in the development and the use of phonological representations, i.e., the abstract representation of speech sounds (e.g., Snowling 2000; but see Ramus \& Szenkovits 2008). Prior to reading, phonological representations consist mainly at the syllable level, and it is only when learning to read that phoneme awareness emerges and explicit phoneme representations develop. Consequently, accurate syllable-level skills are necessary to allow stable phoneme-level representations (e.g., Anthony et al. 2003; Ziegler \& Goswami 2005). Yet, phonological awareness depends strongly on auditory perception skills (McBride-Chang 1996). Within this context, it has been hypothesized that a fundamental deficit in low-level auditory temporal processing underlies the reading and spelling problems in dyslexia (e.g., Tallal 1980; for a review, Farmer \& Klein 1995). Reduced sensitivity to "temporal", i.e., brief and dynamically changing, acoustic cues, the building blocks of spoken language, may result in less specified phonological representations, which in turn leads to a deficiency in reading and spelling skills (McBrideChang 1996).

Adequate temporal processing is one of the most important factors for speech intelligibility. It has been shown that adult listeners are able to accurately perceive speech with only limited spectral information (Shannon et al. 1995). The presence of temporal envelope cues, however, is crucial for speech perception. In continuous speech, the temporal envelope is characterized by multiple rates of amplitude modulations (AM), ranging from 2 to $50 \mathrm{~Hz}$ (Rosen 1992). Particularly slower AM rates, between about 4 and $20 \mathrm{~Hz}$, are important for segmenting speech into smaller units (Drullman et al. 1994; Shannon et al. 1995). Speech segmentation at the lower end of this AM range (near $4 \mathrm{~Hz}$ ) has been found to be important for syllable-level information processing, whereas speech segmentation at the higher end of this range (near $20 \mathrm{~Hz}$ ) provides important phoneme-level information (e.g., formant transitions in stop consonants) (Pöppel 2003).

Several psychophysical studies have investigated AM processing in individuals with dyslexia. In children with dyslexia, elevated AM detection thresholds were observed for modulation frequencies between 4 and $1024 \mathrm{~Hz}$, with the largest discrepancy near $4 \mathrm{~Hz}$, corresponding to the syllabic-rate of speech (Lorenzi et al. 2000; Rocheron et al. 2002). In 
adults with dyslexia, elevated AM detection thresholds were found over a wide range of modulation frequencies between 10 and $320 \mathrm{~Hz}$, indicating AM processing difficulties for phonemic-rate modulation frequencies and for higher modulation frequencies which are less important for speech envelope processing (McAnally et al. 1997; Menell et al. 1999). However, contradicting results were found for AM at the lower end of the speech envelope modulations. Witton et al. (2002) found elevated $20 \mathrm{~Hz}$ AM detection thresholds in dyslexic adults, but observed comparable $2 \mathrm{~Hz}$ AM thresholds between both groups. Stuart et al. (2006) on the other hand, found elevated AM detection thresholds in dyslexic adults in the slow AM range at $1 \mathrm{~Hz}$, but not at $100 \mathrm{~Hz}$.

At the neurophysiological level, less is known about the nature of the temporal processing deficit in dyslexia. Temporal information is coded via neural oscillations that phase-lock to the rhythm of the modulation (Buzsaki \& Draguhn 2004). The ability of the neural system to align temporally to the temporal modulations of the speech envelope, reflected by the phase-locking strength, has been shown to be related to speech comprehension (Ahissar et al. 2001; Luo \& Pöppel 2007). Congruent with psychophysical results, smaller amplitude modulation following responses (AMFR) were observed for AM rates between 10 and $160 \mathrm{~Hz}$, corresponding to phonemic-rate modulations and higher, in dyslexic compared to normal-reading adults (McAnally \& Stein 1997; Menell et al. 1999). Additionally, Abrams et al. (2009) demonstrated a lack of right-hemisphere dominance in temporal brain regions for processing syllabic-rate speech envelope fluctuations in children with dyslexia. Moreover, cortical measures of speech envelope processing predicted up to $41 \%$ of the variability in reading scores and $50 \%$ of phonological awareness, indicating a strong relation between speech envelope processing and reading ability (Abrams et al. 2009). However, not only is the degree of phase-locking important for temporal information processing, also the transmission of the temporal information throughout the auditory system contributes largely to how the temporal envelope of speech is processed. It has been suggested that phase synchronization across spatially distinct brain regions is an important neuronal communication mechanism by dynamically linking neurons into functional networks, which process task-relevant information (Womelsdorf et al. 2007). Phase coherence therefore provides crucial information about functional connectivity in the brain. Reading disability has been related to deviances in phase coherence. Studies found decreased EEG coherences at several EEG bands during rest (Marosi et al. 1995; Shiota et al. 2000; Barry et al. 2009) or during a visuo-spatial attention task (Dhar et al. 2010) in individuals with a reading disability 
(not necessarily dyslexia). However, phase coherence has never been investigated in the auditory domain in a dyslexic population.

In the present study, electrophysiological correlates of AM processing were investigated in a group of normal-reading and dyslexic adults by means of auditory steady state responses (ASSRs). The ASSR is a neurophysiological oscillatory activity, which is typically evoked by amplitude or frequency modulated signals. ASSRs are phase-locked to exactly the same frequency as the modulation and provide an objective measure of the accuracy with which the auditory system processes variations in amplitude and/or frequency (Picton et al. 2003). Although the auditory stimulus activates the entire auditory system, from cochlea to cortex, the frequency of the modulation determines where in the auditory system the electrophysiological response is generated. For AM frequencies near $80 \mathrm{~Hz}$, studies indicate a neural origin at the higher brainstem, whereas the neural origin of the $40 \mathrm{~Hz}$ ASSR has rather been located at both the subcortical and cortical level of the auditory system (John \& Picton 2000; Picton et al. 2003; Bohorquez \& Ozdamar 2008). Less is known about the exact neural sources of ASSRs to low-frequency modulations (below $20 \mathrm{~Hz}$ ). A limited number of ASSR studies reported predominantly primary and/or secondary auditory cortical generators for modulation frequencies lower than $25 \mathrm{~Hz}$ (Rickards \& Clarck 1984; Alaerts et al. 2009). Neuroimaging studies demonstrated that complex spectro-temporal signals like amplitude modulations (AM) and frequency modulations (FM) are processed in the bilateral dorsal superior temporal gyrus (STG) and superior temporal sulcus (STS) (Scott \& Johnsrude 2003). Yet, each hemisphere is specialized in analyzing at a different timescale (Pöppel 2003): slow acoustic modulations between $3-7 \mathrm{~Hz}(150-300 \mathrm{~ms})$ are hypothesized to be processed in the right auditory cortex, whereas the left auditory cortex is thought to be functionally more sensitive to auditory temporal signals between $12-50 \mathrm{~Hz}(20-80 \mathrm{~ms})$. Because accurate AM detection is an essential requisite for precise speech processing, ASSRs to low modulation frequencies may be related to speech perception skills. Studies have shown that ASSRs to modulated tones or noises relate to word recognition scores in adults (Dimitrijevic et al. 2004; Alaerts et al. 2009).

The aim of the present study was to investigate AM processing objectively with multichannel ASSRs in normal-reading and dyslexic adults. By comparing ASSRs to modulation frequencies of 80,20 , and $4 \mathrm{~Hz}$, we aimed to infer whether adults with dyslexia demonstrate AM processing difficulties for all modulation frequencies or whether difficulties are limited to phonemic-rate $(20 \mathrm{~Hz})$ and/or syllabic-rate processing $(4 \mathrm{~Hz})$. Based on findings that phonemic-rate sensitivity builds upon syllabic-rate sensitivity (Ziegler \& Goswami 2005), 
we expected deviant responses at both modulation frequencies in adults with dyslexia. Additionally, the neural system of adults with and without dyslexia will be evaluated in terms of its efficiency to follow the periodicity of these modulations (i.e., response-strength), as well as its ability to functionally connect distant brain regions to process temporal information (i.e., phase coherence). Finally, psychophysical measures of speech-in-noise intelligibility and measures of phonological awareness were administered to examine their relationship with the ASSR measures of neural processing of temporal modulations.

\section{Methods}

\section{Participants}

In this study, 30 normal-reading and 30 dyslexic adults participated (Table 1). Participants were required to have adequate nonverbal intelligence, defined by a standard score above 85 on the Matrices subtest of the "Wechsler Adult Intelligence Scale" (WAIS-III: Wechsler 1992). They were all native Dutch speakers, without a history of brain damage, language problems, psychiatric symptoms, visual problems or hearing loss. All participants were righthanded, assessed by the Edinburgh Handedness Inventory, and were required to have sufficiently good audiometric pure-tone hearing thresholds (i.e., $25 \mathrm{~dB}$ HL or less from 0.25$8.0 \mathrm{kHz}$ ) at the right ear. Participant groups were differentiated based on their history of reading problems and their current reading performance. Participants in the dyslexic group were required: $(i)$ to have a formal diagnosis of developmental dyslexia assessed by a qualified psychologist and (ii) to demonstrate persistent adulthood reading difficulties by scoring below percentile 5 of a university norm group (Depessemier \& Andries 2009) on both a standardized word-reading (Brus \& Voeten 1973) and a pseudoword-reading test (Van den Bos et al. 1994). Participants of the normal-reading group on the other hand: $(i)$ reported no history of reading difficulties; and (ii) scored above percentile 5 of the university norm group at the same standardized word-reading and pseudoword-reading tests. The normal-reading group thus covered the entire range of reading abilities, in the absence of dyslexia. All tests were approved by the Committee of Medical Ethics of Clinical Research of KU Leuven. All participants gave written informed consent. 
Table 1 Participant characteristics $(\mathrm{M} \pm \mathrm{SD})$

\begin{tabular}{lcc}
\hline & Normal-reading $(N=30)$ & Dyslexic $(N=30)$ \\
\hline Gender (f/m) & $20 / 10$ & $22 / 8$ \\
Age (years) & $21.4 \pm 2.7$ & $21.5 \pm 2.7$ \\
Non-verbal IQ & $108 \pm 13$ \\
Word-reading $^{\mathrm{b}}$ & $106 \pm 10$ & $63 \pm 10^{* * *}$ \\
Pseudoword-reading $^{\mathrm{b}}$ & $101 \pm 9$ & $32 \pm 5^{* * *}$ \\
Spelling $^{\mathrm{a}}$ & $63 \pm 10$ & $72 \pm 9^{* * *}$ \\
\hline
\end{tabular}

${ }^{\mathrm{a}}$ Standardized scores with population average $(\mathrm{M}=100, \mathrm{SD}=15)$

${ }^{\mathrm{b}}$ Raw scores (words/min)

$* p<0.05 ; * * p<0.01 ; * * * p<0.001$

\section{Psychophysical measures}

Stimuli of all psychophysical tasks were presented to the right ear with Sennheiser HDA 200 headphones. Phonological awareness was assessed by a phoneme deletion and a spoonerisms task at $70 \mathrm{~dB}$ SPL. In the phoneme deletion task single spoken pseudowords were presented followed by a phoneme. Subjects had to identify the word that remained when that phoneme was omitted from the original word. In the spoonerisms task, two (pseudo)words were presented. Subjects were asked to exchange the two initial letters of both words. Performance of both tasks was assessed by the average response time (in milliseconds) of the correct answers. Response times of both tests were converted to $\mathrm{z}$ scores. The average of $\mathrm{z}$ scores of both tests for the whole group ( $\mathrm{N}=60)$ was calculated as a composite score for phonological awareness. Note that higher composite scores represent inferior phonological awareness performance.

Speech-in-noise intelligibility was assessed for phonemes and sentences. In both tests, the level of the background noise was fixed at $70 \mathrm{~dB}$ SPL, whereas the speech level was varied. Speech and noises were presented with headphones to the right ear. The speech-innoise test for phonemes consisted of CVC words presented in noise. The CVC words were obtained from Flemish recordings of the NVA list, consisting of 15 sublists of 33 target phonemes spoken by a male speaker (Wouters et al. 1994). The stationary NVA speechweighted noise was used as masking noise. The percentage of correct perceived phonemes was measured for SNRs of $-12,-9$ and $-6 \mathrm{~dB}$. For every SNR two lists were administered and performance over both lists was averaged for each SNR. The Speech-Reception-Threshold (SRT) was obtained from a non-linear regression fit to a logistic function of the performance 
of each individual subject. In the speech-in-noise test for sentences, LIST-sentences (spoken by a female speaker) were presented in a stationary speech-weighted noise (van Wieringen $\&$ Wouters 2008). To each subject, two lists of 10 sentences were presented. The SRT was determined adaptively by a one-up, one-down paradigm with a step size of $2 \mathrm{~dB}$. A response was considered correct if all the key words of the sentence were repeated correctly. The SNRs of the last six sentences were averaged to compute the SRT and the SRTs of two subsequent tests were averaged to compute a mean SRT (van Wieringen \& Wouters 2008).

\section{ASSR stimulus parameters}

In this study, continuous amplitude modulated speech-weighted noise stimuli were created. The noise carriers were identical to the LIST noise (van Wieringen \& Wouters 2008). Noises were $100 \%$ amplitude modulated at adjusted modulation frequencies near 4,20 and $80 \mathrm{~Hz}$ (exact frequencies: 3.91, 19.53 and $80.08 \mathrm{~Hz}$ respectively). The modulation frequencies of 4 and $20 \mathrm{~Hz}$ were selected because of their importance in speech intelligibility (Shannon et al. 1995), representing the syllable and phoneme rate respectively. Furthermore, they represent processing at the level of the cortex. The $80 \mathrm{~Hz}$ modulation frequency was selected as a reference condition, to investigate higher modulation frequencies that are less important for speech envelope processing. This modulation frequency also gives information about the integrity of processing at the level of the brainstem (e.g., John \& Picton 2000; Herdman et al. 2002; Picton et al. 2003). Stimuli were generated in Matlab R14. In the experiment, all stimuli were presented with ER-3A insert phones, monaurally to the right ear at $70 \mathrm{~dB}$ SPL.

\section{ASSR recording parameters}

The continuous EEG was recorded by 10 surface $\mathrm{Ag} / \mathrm{AgCl}$ electrodes on the scalp. Recording electrodes were placed in accordance with the international 10-20 system for electrode placement (Malmivuo \& Plonsey 1995), at (1) Oz, (2) P4, (3) P3, (4) F4, (5) F3, (6) M2, i.e., right mastoid (ipsilateral to acoustic stimulation), (7) M1, i.e., left mastoid (contralateral to acoustic stimulation), (8) Fpz, with a reference electrode placed at $\mathrm{Cz}$ and ground electrode at the right clavicle. Inter-electrode impedances were kept below $5 \mathrm{kOhm}$ at $30 \mathrm{~Hz}$. The electrodes were connected to a low-noise Jaeger-Toennies multichannel amplifier. Each EEG channel was amplified with a gain of 50000 and band-pass filtered between 0.2 and $100 \mathrm{~Hz}(6$ dB/octave), 2 and $100 \mathrm{~Hz}$ (6 dB/octave) and 20 and $300 \mathrm{~Hz}$ (6 dB/octave), for the 4, 20 and $80 \mathrm{~Hz}$ modulation frequencies respectively. Because the ASSR is a frequency-specific response, these filter settings only served to minimize the number of artifacts in the EEG. The 
amplified EEG signals were recorded and stimuli were presented by a RME Hammerfall DSP Multiface multichannel soundcard in combination with the experimental Setup ORL for Multichannel ASSR (SOMA) (Van Dun et al. 2008) at a sampling rate of $32 \mathrm{kHz}$ and downsampled to $1 \mathrm{kHz}$. The continuous EEG was recorded in epochs. Each epoch consisted of 1024 samples (corresponding to 1.024 seconds). Sixteen successive epochs were clustered into a sweep. Online artifact rejection was set to $100 \mu \mathrm{V}$, to exclude muscle artifacts. For each stimulus, 18 artifact-free sweeps were recorded. EEG recordings were carried out in a doublewalled and soundproof booth with a Faraday cage. Participants were asked to lie down on a bed and to watch a soundless movie to stay alert. Two ASSR recordings were carried out for each stimulus within the same session.

\section{ASSR data analysis}

Data analyses were performed by means of Matlab R14. Recorded data was divided in epochs. Ten percent of the epochs, i.e., those with the largest noise values, in each recording were rejected to exclude muscle artifacts. Sweeps were constructed by clustering 16 consecutive epochs. For each recording, the remaining 16 sweeps of EEG were weightedaveraged in the time domain and transformed into the frequency domain using a Fast Fourier Transform (FFT). The signal-to-noise ratio (SNR), as a measure of response-strength, was calculated by the log-transformation of the ratio between the response power at the tested modulation frequency and the power estimate of 40 neighbouring frequency bins on each side of the response-frequency bin (corresponding to approximately $2.44 \mathrm{~Hz}$ to the left and right side of the modulation frequency). A response was considered significant when the F-ratio statistic showed a significant difference $(p<0.05)$ between the response-power and the mean noise-power (John \& Picton 2000), corresponding to a response-SNR of $4.85 \mathrm{~dB}$. In the analyses, the baseline level of the response-SNRs was placed at $0 \mathrm{~dB}$ because this indicates that no response can be detected in the EEG noise. Negative response-SNRs were transformed to the baseline level of $0 \mathrm{~dB}$. Including negative response-SNRs in the analyses would falsely give more weight to non-significant responses and would therefore give a too optimistic view on possible group differences. Furthermore, phase coherence was calculated according to the following formula:

$$
C_{12}(f)=\frac{\left|W_{12}(f)\right|^{2}}{\left(W_{11}(f) \times W_{22}(f)\right)}
$$


Phase coherence is a correlation in the time domain between two signals, recorded simultaneously at different scalp electrodes at a given frequency or frequency band. The coherence at frequency $f$ between signals 1 and 2 indicated as $C_{12}(f)$ is calculated as the squared magnitude of the cross-spectral density of the signal pair $W_{12}(f)$, normalized by dividing with the product of the power spectra of the two signals, respectively $W_{11}(f)$ and $W_{22}(f)$ (Nunez \& Srinivasan 2006). Coherence values range between zero and one. Low coherence indicates no synchronization between underlying brain regions, whereas high coherence indicates a high degree of synchronized neuronal activity.

\section{Statistical analyses}

Statistical analyses were performed with the software SPSS 16 (SPSS 2007). For each stimulus, a test and retest were recorded. Because there were no significant differences in response-SNR between the test and retest (paired t-test, $|t|<1.99, p>0.05$ for all electrodes and modulation frequencies), both recordings were linked together and FFT statistics were carried out over the linked data, i.e., after 32 sweeps. This prolonged duration resulted in better averaging of the data and higher response-SNRs. Normality of the response-SNRs and coherences was tested by a Kolmogorov-Smirnov test separately for all groups, modulation frequencies and electrodes. The significance level was set at $p<0.01$, to adjust for multiple comparisons. All response-SNRs and coherence values were normally distributed according to this criterion.

Table 2 Average response-Signal-to-Noise-Ratios (response-SNRs) and percentage of subjects with nonsignificant responses for frontal, mastoid and parietal electrodes for modulation frequencies of 80,20 and $4 \mathrm{~Hz}$

\begin{tabular}{lcccccc}
\hline & \multicolumn{3}{c}{ response-SNR (dB) } & \multicolumn{2}{c}{$\%$ nonsignificant responses } \\
\hline & frontal & Mastoid & parietal & frontal & mastoid & parietal \\
\hline $\mathbf{8 0 ~ H z}$ & 10 & 16 & 17 & $22 \%$ & $3 \%$ & $4 \%$ \\
$\mathbf{2 0 ~ H z}$ & 9 & 18 & 15 & $21 \%$ & $3 \%$ & $8 \%$ \\
$\mathbf{4 ~ H z}$ & 7 & 12 & 11 & $27 \%$ & $6 \%$ & $14 \%$ \\
\hline
\end{tabular}


Some electrode positions were more sensitive in recording responses than others were. For the three modulation frequencies the highest response-SNRs were found at mastoid (M1, M2), parietal $(\mathrm{P} 3, \mathrm{P} 4)$ and occipital $(\mathrm{Oz})$ electrodes, whereas the smallest responses were recorded at frontal electrodes (F3, F4, Fpz). Additionally, at these frontal electrodes it was more difficult to record significant responses as evidenced by the higher number of subjects with nonsignificant responses (Table 2). Nonsignificant responses only indicate that a response is statistically not present but do not provide any quantitative information. Therefore, responseSNRs and coherence values of parietal and mastoid electrodes seem to be the most reliable measures for neural processing at the three tested modulation frequencies. To allow the comparison of right and left hemisphere responses, the midline occipital electrode $(\mathrm{Oz})$ was excluded from the analyses. Summarizing, in the statistical analyses we focused on lateral parietal (P3, P4) and mastoid (M1, M2) electrodes. For these electrodes, response-SNRs and intrahemispheric (right: P4M2; left: P3M1) and interhemispheric coherence (parietal: P3P4; mastoid: M1M2) were evaluated (Figure 1). Note that in these analyses both significant and nonsignificant response-SNRs were included to also account for the worst performances.

In this study, group differences were assessed by a series of repeated measures analyses of variance (RM-ANOVA). All statistical tests were two-tailed $(\alpha=0.05)$. Reported p-values from the RM-ANOVA were Greenhouse-Geisser corrected and post-hoc analyses were Bonferroni corrected for multiple comparisons. Pearson correlations were calculated to determine the relationship between ASSR measures and measures of phonological awareness and speech-in-noise intelligibility.

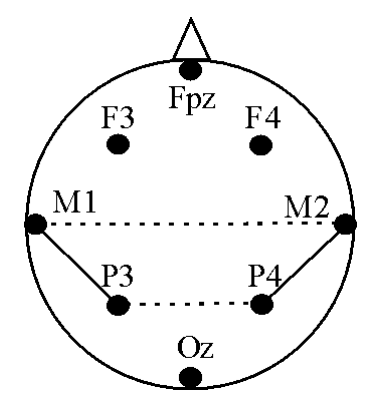

Figure 1 Schematic view of electrode positions and calculated coherence measures. Interhemispheric coherences are shown by dashed lines (M1M2, P3P4), intrahemispheric coherences are depicted in full lines (P4M2, P3M1). 


\section{Results}

\section{Response-strength}

For the three tested modulation frequencies, a RM-ANOVA with factors Group (normalreading vs. dyslexic), Laterality (right vs. left hemisphere) and Electrode (mastoid vs. parietal) was conducted.

For $80 \mathrm{~Hz}$, no main effect of Electrode $\left(F(1,58)=0.60, p=0.440, \eta_{p}{ }^{2}=0.01\right)$, Group $\left(F(1,58)=1.87, p=0.177, \eta_{p}{ }^{2}=0.03\right.$ ) or any interaction with these factors (all $F<0.41, p>$ 0.5) was observed, suggesting no group difference in response-strength for the $80 \mathrm{~Hz}$ ASSR. Overall, a main effect of Laterality $\left(F(1,58)=44.03, p<0.001, \eta_{p}{ }^{2}=0.43\right)$ was found, demonstrating that ipsilateral right hemisphere responses were higher than contralateral left hemisphere responses (mean difference: $3.5 \mathrm{~dB}$, SE: $0.5 \mathrm{~dB}$ ) (Figure 2A).

For $20 \mathrm{~Hz}$, no main effect of $\operatorname{Group}\left(F(1,58)=2.35, p=0.131, \eta_{p}{ }^{2}=0.04\right)$, but significant main effects of Laterality $\left(F(1,58)=15.95, p<0.001, \eta_{p}{ }^{2}=0.22\right)$ and Electrode $\left(F(1,58)=56.48, p<0.001, \eta_{p}{ }^{2}=0.49\right)$ were found. Additionally, a significant Laterality $\mathrm{x}$ Group $\left(F(1,58)=4.36, p=0.041, \eta_{p}^{2}=0.07\right)$ interaction was observed. Post hoc analysis indicated that dyslexic adults had lower responses at left hemisphere electrodes than normalreading adults (mean difference: $2.7 \mathrm{~dB}$, SE: $1.3 \mathrm{~dB}, t(58)=2.04, p=0.046, \eta_{p}{ }^{2}=0.07$ ), whereas this group difference was not present for right hemisphere electrodes (mean difference: $\left.1.1 \mathrm{~dB}, \mathrm{SE}: 1.3 \mathrm{~dB}, t(58)=0.85, p=0.397, \eta_{p}{ }^{2}=0.01\right)$ (Figure 2B).

For $4 \mathrm{~Hz}$, no main effect of $\operatorname{Group}\left(F(1,58)=0.77, p=0.385, \eta_{p}{ }^{2}=0.01\right)$ or any interaction with this factor (all $F<2.45, p>0.1$ ) was found, suggesting no group differences for this modulation frequency (Figure 2C). Overall, a main effect of Laterality $(F(1,58)=$ $\left.12.43, p=0.001, \eta_{p}{ }^{2}=0.18\right)$ and Electrode $\left(F(1,58)=20.35, p<0.001 ; \eta_{p}{ }^{2}=0.26\right)$, and a significant Laterality $\mathrm{x}$ Electrode interaction $\left(F(1,58)=8.37, p=0.005 ; \eta_{p}{ }^{2}=0.13\right)$ was found. At parietal electrodes, ipsilateral right hemisphere responses were higher than contralateral left hemisphere responses (mean difference: $2.0 \mathrm{~dB}, \mathrm{SE}: 0.5 \mathrm{~dB}, t(59)=3.72, p<$ $\left.0.001, \eta_{p}{ }^{2}=0.19\right)$, whereas this hemispheric difference was not present for mastoid electrodes (mean difference: $0.4 \mathrm{~dB}$, SE: $0.3 \mathrm{~dB}, t(59)=1.23, p=0.226, \eta_{p}{ }^{2}=0.03$ ). 


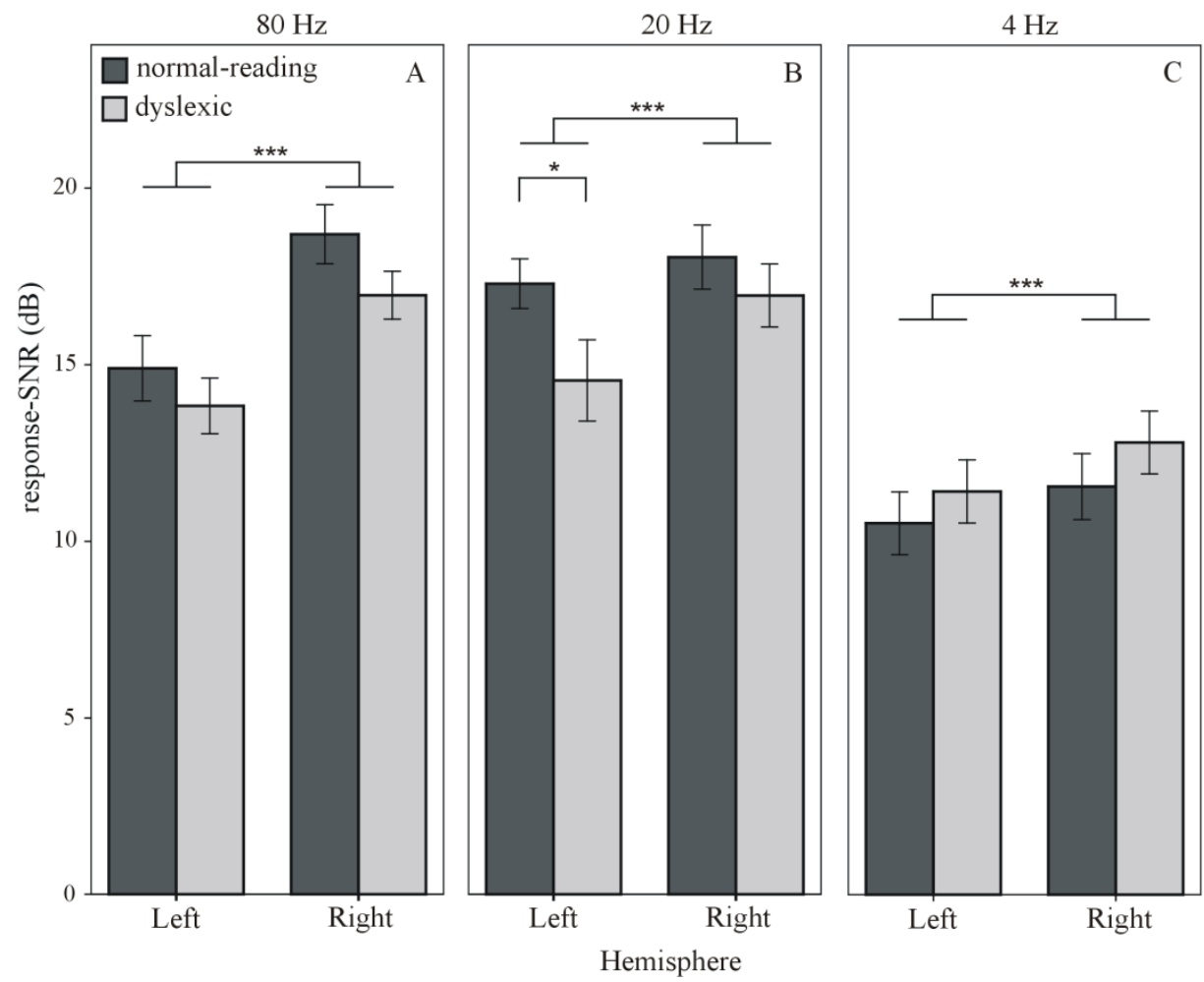

Figure 2 Average response-strengths for normal-reading (dark grey) and dyslexic (light grey) adults, averaged over mastoid and parietal electrodes, for three modulation frequencies. Bargraphs for (A) brainstem processing ( $80 \mathrm{~Hz}$ AM), (B) cortical phonemic-rate processing (20 Hz AM) and (C) cortical syllabic-rate processing (4 Hz AM). Bars represent the average response-SNRs for left and right hemisphere electrodes. Error bars indicate \pm 1 SE. $* p \leq 0.05$, $* * p \leq 0.01, * * * p \leq 0.001$ 


\section{Coherence}

Interhemispheric coherence was analyzed by a RM-ANOVA with factors Group (normalreading vs. dyslexic) and Position (mastoid (M1M2) vs. parietal (P3P4)).

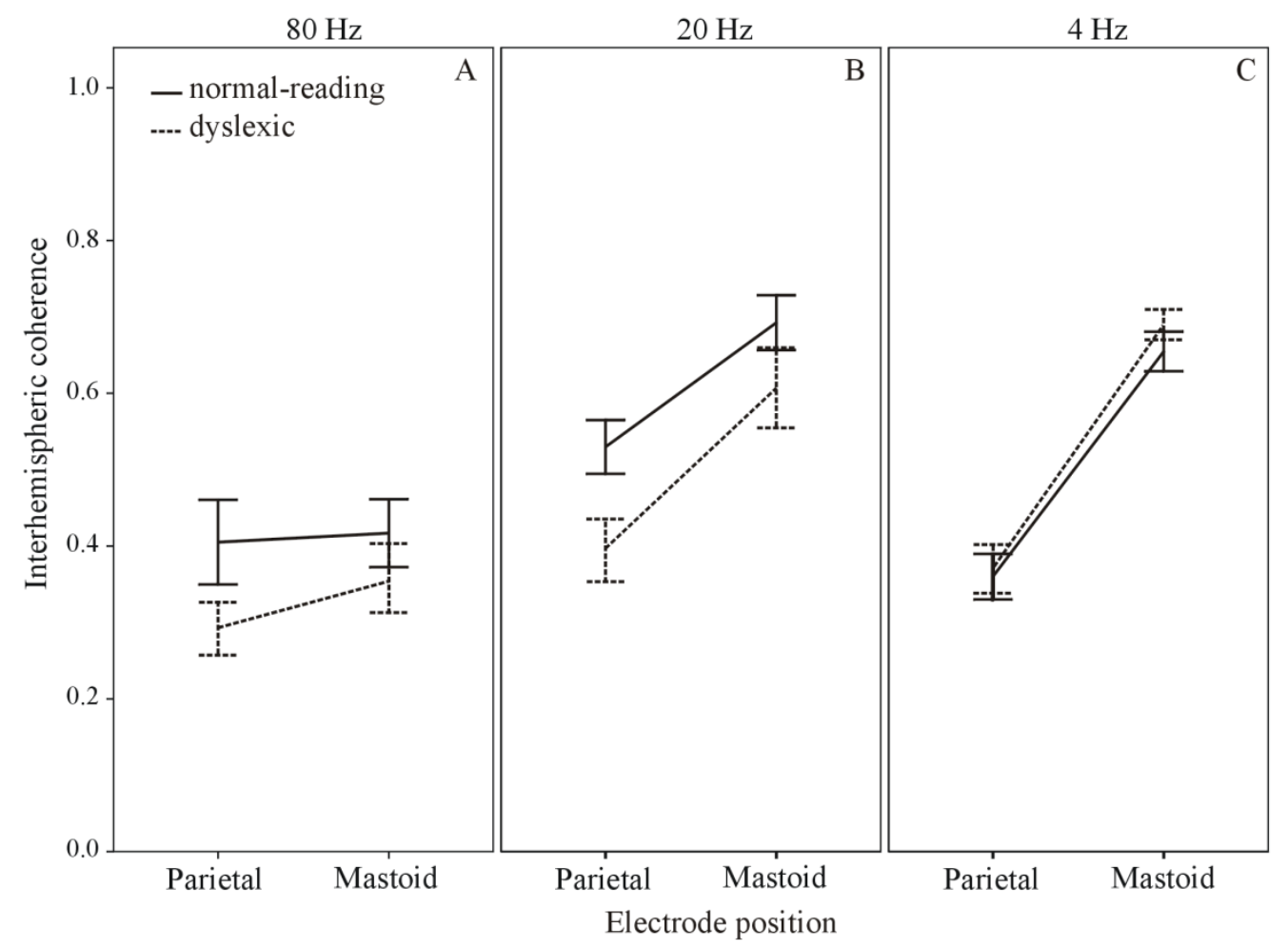

Figure 3 Interhemispheric phase coherence. Interhemispheric phase coherence for normalreading (full lines) and dyslexic (dashed lines) adults for parietal and mastoid electrodes for (A) $80 \mathrm{~Hz} \mathrm{AM,} \mathrm{(B)} 20 \mathrm{~Hz} \mathrm{AM}$ and (C) $4 \mathrm{~Hz}$ AM. Error bars indicate $\pm 1 \mathrm{SE}$.

For $80 \mathrm{~Hz}$, no main effects of $\operatorname{Group}\left(F(1,58)=2.85, p=0.097, \eta_{p}^{2}=0.05\right)$ or Position $\left(F(1,58)=1.01, p=0.319, \eta_{p}{ }^{2}=0.02\right)$, nor a significant Group x Position interaction $(F(1,58)$ $=0.49, p=0.486, \eta_{p}{ }^{2}=0.01$ ) was found, indicating no group differences for the $80 \mathrm{~Hz}$ ASSR (Figure 3A).

On the other hand for $20 \mathrm{~Hz}$, a significant main effect of Group $(F(1,58)=4.42, p=$ $\left.0.040, \eta_{p}{ }^{2}=0.07\right)$, and Position $\left(F(1,58)=52.03, p<0.001, \eta_{p}{ }^{2}=0.47\right)$, but no significant Group x Position interaction $\left(F(1,58)=0.87, p=0.356, \eta_{p}{ }^{2}=0.02\right)$ was found. Overall, interhemispheric coherence at mastoid electrodes was larger than coherence at parietal electrodes (mean difference: 0.19, SE: 0.03). Additionally, normal-reading adults demonstrated higher interhemispheric coherence compared to adults with dyslexia (mean difference: 0.11, SE: 0.05) (Figure 3B). 
For $4 \mathrm{~Hz}$, a main effect of Position $\left(F(1,58)=310.24, p<0.001, \eta_{p}{ }^{2}=0.84\right)$ was found. However, no main effect of $\operatorname{Group}\left(F(1,58)=0.43, p=0.516, \eta_{p}{ }^{2}=0.01\right)$ nor a Group $\mathrm{x}$ Position interaction $\left(F(1,58)=0.51, p=0.480, \eta_{p}^{2}=0.01\right)$ was found, indicating no group differences for the $4 \mathrm{~Hz}$ ASSR (Figure 3C). Overall, coherence at mastoid electrodes was larger than coherence at parietal electrodes (mean difference: 0.31, SE: 0.02).

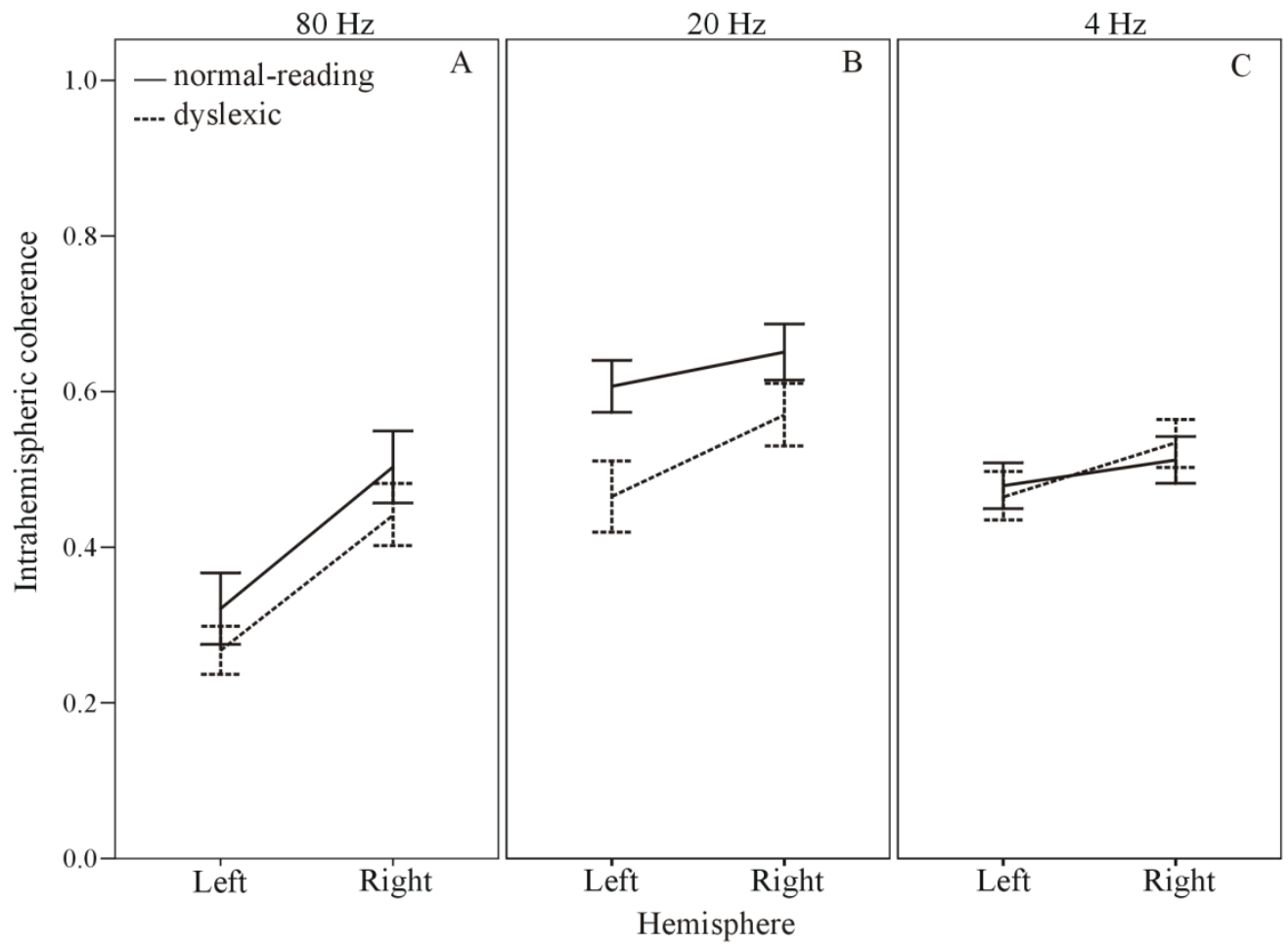

Figure 4 Intrahemispheric phase coherence. Intrahemispheric phase coherence for normalreading (full lines) and dyslexic (dashed lines) adults for left and right hemisphere electrodes for (A) $80 \mathrm{~Hz} \mathrm{AM,} \mathrm{(B)} 20 \mathrm{~Hz} \mathrm{AM}$ and (C) $4 \mathrm{~Hz}$ AM. Error bars indicate $\pm 1 \mathrm{SE}$.

Additionally, intrahemispheric coherence was analyzed by RM-ANOVA with the factors Group (normal-reading vs. dyslexic) and Laterality (right (P4M2) vs. left (P3M1) hemisphere).

For $80 \mathrm{~Hz}$, a main effect of Laterality was found $\left(F(1,58)=39.50, p<0.001, \eta_{p}{ }^{2}=\right.$ 0.41). No main effect of Group $\left(F(1,58)=1.27, p=0.265, \eta_{p}{ }^{2}=0.02\right)$, nor a Group $\mathrm{x}$ Laterality interaction was observed $\left(F(1,58)=0.02, p=0.893, \eta_{p}{ }^{2}<0.01\right)$, indicating no group differences for this modulation frequency (Figure 4A). In general, right hemisphere coherence was higher than left hemisphere coherence (mean difference: 0.18, SE: 0.03). 
For $20 \mathrm{~Hz}$, a main effect of Group $\left(F(1,58)=4.42, p=0.040, \eta_{p}{ }^{2}=0.07\right)$ and Laterality $\left(F(1,58)=20.91, p<0.001, \eta_{p}{ }^{2}=0.27\right)$, but no Group x Laterality interaction $\left.F(1,58)=3.53, p=0.065, \eta_{p}{ }^{2}=0.06\right)$ was found. Coherence in the right hemisphere was higher than coherence in the left hemisphere (mean difference: 0.08, SE: 0.02). Additionally, adults with dyslexia demonstrated lower intrahemispheric coherence than normal-reading adults (mean difference: 0.11, SE: 0.05) (Figure 4B).

For $4 \mathrm{~Hz}$, a main effect of Laterality $\left(F(1,58)=7.23, p=0.009, \eta_{p}^{2}=0.11\right)$ was observed. No main effect of Group $\left(F(1,58)=0.01, p=0.913, \eta_{p}{ }^{2}<0.01\right)$, nor a significant Group x Laterality interaction $\left(F(1,58)=0.84, p=0.364, \eta_{p}{ }^{2}=0.01\right)$ was found, indicating no group differences for this modulation frequency (Figure 4C). Overall, coherence in the right hemisphere was higher than coherence in the left hemisphere (mean difference: 0.05, SE: $0.02)$.

\section{Correlations}

Pearson correlations were calculated to examine relations between ASSR coherence and psychophysical measures of speech-in-noise intelligibility and phonological awareness (Table 3). Better phonological awareness skills related to higher coherence at $20 \mathrm{~Hz}$ but not at $4 \mathrm{~Hz}$ or $80 \mathrm{~Hz}$. Furthermore, sentence speech-in-noise intelligibility correlated significantly with 4 $\mathrm{Hz}$, but not with $20 \mathrm{~Hz}$ ASSR coherence. Additionally, phoneme-level speech-in-noise intelligibility correlated with ASSR coherence at $20 \mathrm{~Hz}$ but not with coherence at $4 \mathrm{~Hz}$. For speech-in-noise perception, higher phonemic-rate coherence results in better phoneme-level speech-in-noise intelligibility, whereas higher syllabic-rate coherence results in better sentence-level speech-in-noise intelligibility. This was not the case for $80 \mathrm{~Hz}$, where only right intrahemispheric coherence correlated with speech-in-noise perception for sentences. Comparable results were also obtained for correlations between response-SNRs and psychophysical measures for speech-in-noise intelligibility and phonological awareness. However, these correlations, especially those with phonological awareness, were smaller and often failed to reach significance. 
Table 3 Whole-sample correlations between ASSR coherence at 80, 20 and $4 \mathrm{~Hz}$, and phonological awareness (PA) and speech-in-noise intelligibility (SPIN)

\begin{tabular}{|c|c|c|c|c|c|c|c|c|c|c|c|c|}
\hline & \multicolumn{4}{|c|}{$80 \mathrm{~Hz}$} & \multicolumn{4}{|c|}{$20 \mathrm{~Hz}$} & \multicolumn{4}{|c|}{$4 \mathrm{~Hz}$} \\
\hline & \multicolumn{2}{|c|}{ INTER } & \multicolumn{2}{|c|}{ INTRA } & \multicolumn{2}{|c|}{ INTER } & \multicolumn{2}{|c|}{ INTRA } & \multicolumn{2}{|c|}{ INTER } & \multicolumn{2}{|c|}{ INTRA } \\
\hline & parietal & mastoid & left & right & parietal & mastoid & left & right & parietal & mastoid & left & right \\
\hline $\mathrm{PA}^{\mathrm{a}}$ & -0.22 & -0.06 & -0.16 & -0.16 & $-0.36 * *$ & -0.18 & $-0.33 * *$ & $-0.27 *$ & 0.13 & 0.22 & 0.10 & 0.02 \\
\hline SPIN $^{\mathrm{b}}$ (sentences) & -0.15 & -0.15 & -0.18 & $-0.26^{*}$ & 0.09 & -0.04 & 0.03 & 0.07 & $-0.26 *$ & -0.11 & $-0.27 *$ & -0.10 \\
\hline
\end{tabular}

${ }^{\mathrm{a} C}$ Composite phonological awareness score of response times

${ }^{\mathrm{b}}$ Speech-Reception-Threshold (SRT)

$* p<0.05 ; * * p<0.01$ 


\section{Discussion}

Identifying the neural foundations of reading and spelling problems in dyslexia is an important aspect for early detection and appropriate intervention of children with this disability. It has been hypothesized that the literacy problems in dyslexia can be allocated to deviant mechanisms for auditory temporal modulation processing that are critical for accurate speech perception and subsequently also for precise development of phonological representations. The present study demonstrated with objective measures that adults with dyslexia have difficulties processing phonemic-rate modulations and that these difficulties are related to reduced speech perception and phonological skills. The present study recorded ASSRs in a population of adults with dyslexia. ASSRs provide an objective measure for auditory temporal modulation processing in that they are elicited automatically without cognitive demand, given that the subject is in a state of alertness (Picton et al. 2003). More importantly, the presentation of modulated steady state stimuli allows objective response detection in the EEG frequency spectrum, exactly at the presented modulation frequency. By comparing responses to several modulation frequencies, we were able to map phase-locking efficiency at important levels of the auditory system and at several modulation frequencies that are critical for phonological development.

Adults with dyslexia demonstrated normal temporal modulation processing for the 80 $\mathrm{Hz}$ amplitude modulation for response-strength, coherence and lateralization. A number of studies investigated the neural origin of this ASSR. As one ascends the auditory pathway from auditory nerve until the cortex, neurons become less sensitive in following high modulation rates (Picton et al. 2003). Magnetoencephalography (MEG) studies identified $80 \mathrm{~Hz}$ ASSR sources in the brainstem (Herdman et al. 2002). Additionally, EEG studies found latencies between 10-30 ms for the $80 \mathrm{~Hz}$ ASSR, corresponding to neural sources in the higher brainstem that may in part be accompanied with early cortical sources (e.g., John \& Picton 2000; Picton et al. 2003). Yet, the finding that $80 \mathrm{~Hz}$ ASSRs are not influenced by sleep or anesthesia (Cohen et al. 1991; Aoyagi et al. 1993) argues indirectly for minimal cortical contribution in the generation of this response. The present results suggest that in adults with dyslexia sensitivity to those temporal modulations that are generated in the brainstem is normal. These results seem to contradict studies demonstrating deviant speech-evoked ABRs in children with reading disabilities (e.g., Wible et al. 2004; Banai et al. 2005; Wible et al. 2005; Banai et al. 2009; Hornickel et al. 2009; Hornickel et al. 2011). However, most studies demonstrating the link between speech-evoked ABRs and reading abilities included a broad variety of language disorders other than dyslexia (e.g., poor readers, specific language 
disorder). As poor reading abilities can result from a variety of underlying cognitive and neurological problems, it would be incorrect to generalize findings of atypical speech-evoked ABRs to a specific population of individuals with dyslexia. Additionally, speech-evoked ABRs and ASSRs are evoked by distinct acoustic features. Whereas speech-evoked ABRs encode the onset/offset and the fine structure of an auditory signal (e.g., Skoe \& Kraus 2010), ASSRs follow the ongoing modulation envelope of the signal (e.g., Picton et al. 2003). Both measures are therefore rather complementary than contradictory.

Normal-reading adults and adults with dyslexia differed in their ability to phase-lock to the phonemic processing rate $(20 \mathrm{~Hz})$. This difference was observed in response-strengths as well as in intrahemispheric and interhemispheric coherence. Neurophysiologically, the strength of an ASSR depends on the phase-locking ability of neural populations to the temporal envelope of a stimulus. Decreased response-strengths could therefore result from either a reduced number of neurons that phase-lock to modulations or alternatively from asynchronicity of neuronal firing within a neural generator. On the other hand, reduced phase coherence between electrodes implies decreased or imprecise synchronization between distant neuronal ensembles, suggesting decreased information transfer between distant brain regions involved in the same process. Even though the present study cannot make conclusions about impairments at the neuronal level, it is reasonable to conclude that the observed $20 \mathrm{~Hz}$ results reflect a general neurological deviance in processing these speech envelope cues in adults with dyslexia. Decreased brain activity and reduced functional connectivity in dyslexia has previously been reported, however this has never been directly linked to speech envelope processing. During word and pseudoword reading tasks, a number of studies found decreased brain activity in individuals with dyslexia, especially in the left hemisphere (e.g., Shaywitz 1998; Pugh et al. 2000). Additionally, Diffusion Tensor Imaging (DTI) studies have provided evidence for a decreased white matter integrity (i.e., less efficient neural communication) in the left temporo-parietal cortex in dyslexic individuals (Horwitz et al. 1998; Pugh et al. 2000), and more specifically in the left arcuate fasciculus (AF) and superior longitudinal fasciculus (SLF) (e.g., Klingberg et al. 2000; Rimrodt et al. 2010). Both white matter tracts are part of a left hemisphere dorsal pathway, responsible for mapping phonological representations onto articulatory representations. If the functional integrity of dorsal sound-to-articulation pathway in the left hemisphere indeed underlies the reading problems in dyslexia, it is possible that this deficit stems from an earlier problem at the acoustic-phonological level, i.e., in the processing of the speech envelope. Moreover, to accurately map sounds to articulatory units, acoustic signals need to be clustered into phonological sound units. 
Group differences were observed for the phonemic processing rate $(20 \mathrm{~Hz})$, but not for the syllabic-rate $(4 \mathrm{~Hz})$. Comparable to the present findings, previous studies demonstrated reduced AM sensitivity at the phonemic-rate (McAnally \& Stein 1997; Menell et al. 1999) but not at the syllabic-rate in adults with dyslexia (Witton et al. 2002). However, in children with dyslexia, both syllabic and phonemic-rate processing difficulties have been evidenced (Lorenzi et al. 2000; Rocheron et al. 2002; Abrams et al. 2009). These studies suggest that temporal processing difficulties in individuals with dyslexia occur at the same level of the individual's phonological development. Assuming that phonological awareness builds upon accurate encoding of spoken language, which in turn is influenced by the ability to phase-lock to temporal modulations, it is possible that sensitivity for specific modulations develops in a similar way as phonological development. Indeed, in the present study, phonological awareness related significantly to the $20 \mathrm{~Hz}$ ASSR and not to the $4 \mathrm{~Hz}$ ASSR. Additionally, ASSRs to 20 and $4 \mathrm{~Hz}$ AM related significantly to phoneme and sentence in noise perception respectively, indicating the link between sensitivity for these modulations and speech perception. If sensitivity to syllabic-rate and phonemic-rate modulations indeed relates to the grain size of phonological awareness, one may expect that children that later develop dyslexia demonstrate AM processing difficulties at the syllabic-rate prior to reading instruction, whereas phonemic-rate difficulties rather appear for experienced readers. However, this hypothesis has never been investigated directly. If a developmental syllabic-to-phonemic-rate shift truly exists, one may expect that in children a syllabic-rate deficiency will be more pronounced compared to adults. To clarify this hypothesis, studies investigating syllabic and phonemic-rate processing in children of different age groups are necessary. Moreover, to enlighten the development of these temporal modulations and to clarify their influence on literacy development, longitudinal studies including children at-risk for dyslexia are required.

The present results bring new light on the discussion as to whether an auditory temporal processing deficit, causing phonological and literacy problems, exists in dyslexia. Dyslexic adults seem to have a general neurological disability to process phonemic-rate modulations in speech, whereas syllabic-rate modulation processing seems to be intact. Even though we cannot make conclusions about causality of phonemic-rate phase-locking and speech perception, phonological and literacy skills, we found evidence for a relation between these abilities. Further research in a population of children with dyslexia is necessary to unravel this causal relationship and to validate whether the hypothesized developmental shift from syllabic-rate to phonemic-rate sensitivity actually occurs. 


\section{Acknowledgements}

The research was financed by the fund for Scientific Research Flanders, grant G.0331.08 and a grant of the Research Council of Katholieke Universiteit Leuven, OT/07/034. We are grateful to all participants. Special thanks are due to M. Hofmann for technical assistance and to A. Van Bogaert for assistance in data acquisition.

\section{References}

Abrams, D. A., Nicol, T., Zecker, S. et al. (2009). Abnormal cortical processing of the syllable rate of speech in poor readers. J Neurosci, 29, 7686-7693.

Ahissar, E., Nagarajan, S., Ahissar, M. et al. (2001). Speech comprehension is correlated with temporal response patterns recorded from auditory cortex. Proc Natl Acad Sci U S A, 98, 13367-13372.

Alaerts, J., Luts, H., Hofmann, M. et al. (2009). Cortical auditory steady-state responses to low modulation rates. Int J Audiol, 48, 582-593.

Anthony, J. L., Lonigan, C. J., Driscoll, K. et al. (2003). Phonological sensitivity: A quasiparallel progression of word structure units and cognitive operations. Reading Research Quarterly, 38, 470-487.

Aoyagi, M., Kiren, T., Kim, Y. et al. (1993). Optimal Modulation Frequency for AmplitudeModulation Following Response in Young-Children During Sleep. Hearing Research, 65, 253-261.

Banai, K., Hornickel, J., Skoe, E. et al. (2009). Reading and subcortical auditory function. Cereb Cortex, 19, 2699-2707.

Banai, K., Nicol, T., Zecker, S. G. et al. (2005). Brainstem timing: Implications for cortical processing and literacy. J Neurosci, 25, 9850-9857.

Barry, R. J., Clarke, A. R., McCarthy, R. et al. (2009). EEG coherence in children with attention-deficit/hyperactivity disorder and comorbid reading disabilities. Int J Psychophysiol, $71,205-210$.

Bohorquez, J. \& Ozdamar, O. (2008). Generation of the 40-Hz auditory steady-state response (ASSR) explained using convolution. Clin Neurophysiol, 119, 2598-2607.

Brus, B. T. \& Voeten, M. J. M. (1973). Een minuut test, vorm A en B. Nijmegen, The Netherlands: Berkhout.

Buzsaki, G. \& Draguhn, A. (2004). Neuronal oscillations in cortical networks. Science, 304, 1926-1929.

Cohen, L. T., Rickards, F. W., \& Clark, G. M. (1991). A Comparison of Steady-State EvokedPotentials to Modulated Tones in Awake and Sleeping Humans. Journal of the Acoustical Society of America, 90, 2467-2479. 
Depessemier, P. \& Andries, C. (2009). Gletschr, test voor gevorderd lezen en schrijven. Antwerp, Belgium: Garant.

Dhar, M., Been, P. H., Minderaa, R. B. et al. (2010). Reduced interhemispheric coherence in dyslexic adults. Cortex, 46, 794-798.

Dimitrijevic, A., John, M. S., \& Picton, T. W. (2004). Auditory steady-state responses and word recognition scores in normal-hearing and hearing-impaired adults. Ear Hear, 25, 68-84.

Drullman, R., Festen, J. M., \& Plomp, R. (1994). Effect of reducing slow temporal modulations on speech reception. J Acoust Soc Am, 95, 2670-2680.

Farmer, M. E. \& Klein, R. M. (1995). The evidence for a temporal processing deficit linked to dyslexia: A Review. Psychon Bull Rev, 2, 460-493.

Herdman, A. T., Lins, O., Van Roon, P. et al. (2002). Intracerebral sources of human auditory steady-state responses. Brain Topogr, 15, 69-86.

Hornickel, J., Skoe, E., Nicol, T. et al. (2009). Subcortical differentiation of stop consonants relates to reading and speech-in-noise perception. Proc Natl Acad Sci U S A, 106, 1302213027.

Hornickel, J., Chandrasekaran, B., Zecker, S. et al. (2011). Auditory brainstem measures predict reading and speech-in-noise perception in school-aged children. Behav Brain Res, 216, 597-605.

Horwitz, B., Rumsey, J. M., \& Donohue, B. C. (1998). Functional connectivity of the angular gyrus in normal reading and dyslexia. Proc Natl Acad Sci U S A, 95, 8939-8944.

John, M. S. \& Picton, T. W. (2000). Human auditory steady-state responses to amplitudemodulated tones: phase and latency measurements. Hear Res, 141, 57-79.

Klingberg, T., Hedehus, M., Temple, E. et al. (2000). Microstructure of temporo-parietal white matter as a basis for reading ability: Evidence from diffusion tensor magnetic resonance imaging. Neuron, 25, 493-500.

Lorenzi, C., Dumont, A., \& Fullgrabe, C. (2000). Use of temporal envelope cues by children with developmental dyslexia. J Speech Lang Hear Res, 43, 1367-1379.

Luo, H. \& Pöppel, D. (2007). Phase patterns of neuronal responses reliably discriminate speech in human auditory cortex. Neuron, 54, 1001-1010.

Malmivuo, J. \& Plonsey, R. (1995). Bioelectromagnetism: Principles and applications of bioelectric and biomagnetic fields. New York, USA: Oxford University Press.

Marosi, E., Harmony, T., Becker, J. et al. (1995). Electroencephalographic coherences discriminate between children with different pedagogical evaluation. Int J Psychophysiol, 19, 23-32.

McAnally, K. I., Hansen, P. C., Cornelissen, P. L. et al. (1997). Effect of time and frequency manipulation on syllable perception in developmental dyslexics. J Speech Lang Hear Res, 40, 912-924. 
McAnally, K. I. \& Stein, J. F. (1997). Scalp potentials evoked by amplitude-modulated tones in dyslexia. J Speech Lang Hear Res, 40, 939-945.

McBride-Chang, C. (1996). Models of speech perception and phonological processing in reading. Child Dev, 67, 1836-1856.

Menell, P., McAnally, K. I., \& Stein, J. F. (1999). Psychophysical sensitivity and physiological response to amplitude modulation in adult dyslexic listeners. J Speech Lang Hear Res, 42, 797-803.

Nunez, P. L. \& Srinivasan, R. (2006). Electric fields of the brain: The neurophysics of EEG. (2nd ed.) New York, USA: Oxford University Press.

Picton, T. W., John, M. S., Dimitrijevic, A. et al. (2003). Human auditory steady-state responses. Int J Audiol, 42, 177-219.

Pöppel, D. (2003). The analysis of speech in different temporal integration windows: cerebral lateralization as 'asymmetric sampling in time'. Speech Commun, 41, 245-255.

Pugh, K. R., Mencl, W. E., Jenner, A. R. et al. (2000). Functional neuroimaging studies of reading and reading disability (developmental dyslexia). Ment Retard Dev Disabil Res Rev, 6, 207-213.

Ramus, F. \& Szenkovits, G. (2008). What phonological deficit? The Quarterly Journal of Experimental Psychology, 61, 129-141.

Rickards, F. W. \& Clarck, G. M. (1984). Steady-state evoked potentials to amplitudemodulated tones. In R.H.Nodar \& C. Barber (Eds.), Evoked potentials II (pp. 163-168). Boston, USA: Butterworth.

Rimrodt, S. L., Peterson, D. J., Denckla, M. B. et al. (2010). White matter microstructural differences linked to left perisylvian language network in children with dyslexia. Cortex, 46, 739-749.

Rocheron, I., Lorenzi, C., Fullgrabe, C. et al. (2002). Temporal envelope perception in dyslexic children. Neuroreport, 13, 1683-1687.

Rosen, S. (1992). Temporal information in speech: acoustic, auditory and linguistic aspects. Philos Trans R Soc Lond B Biol Sci, 336, 367-373.

Scott, S. K. \& Johnsrude, I. S. (2003). The neuroanatomical and functional organization of speech perception. Trends Neurosci, 26, 100-107.

Shannon, R. V., Zeng, F. G., Kamath, V. et al. (1995). Speech recognition with primarily temporal cues. Science, 270, 303-304.

Shaywitz, B. A. (1998). Functional organization of the brain of reading and dyslexia. Biol Psychiatry, 43, 210.

Shiota, M., Koeda, T., \& Takeshita, K. (2000). Cognitive and neurophysiological evaluation of Japanese dyslexia. Brain \& Development, 22, 421-426. 
Skoe, E. \& Kraus, N. (2010). Auditory brain stem response to complex sounds: A tutorial. Ear Hear, 31, 302-324.

Snowling, M. (2000). Dyslexia. (2nd ed.) Oxford, UK: Blackwell.

SPSS (2007): Statistical Package Social Science (SPSS). Chicago, IL: SPSS Inc.

Stuart, G. W., McAnally, K. I., Mckay, A. et al. (2006). A test of the magnocellular deficit theory of dyslexia in an adult sample. Cogn Neuropsychol, 23, 1215-1229.

Tallal, P. (1980). Auditory temporal perception, phonics, and reading disabilities in children. Brain Lang, 9, 182-198.

Van den Bos, K. P., Spelberg, H. C. L., Scheepstra, A. J. M., \& De Vries, J. R. (1994). De Klepel. Vorm A en B. Een test voor de leesvaardigheid van pseudowoorden. Verantwoording, handleiding, diagnostiek en behandeling. Nijmegen, The Netherlands: Berkhout.

Van Dun, B., Verstraeten, S., Alaerts, J. et al. (2008). A flexible research platform for multichannel auditory steady-state response measurements. J Neurosci Methods, 169, 239-248.

van Wieringen, A. \& Wouters, J. (2008). LIST and LINT: Sentences and numbers for quantifying speech understanding in severely impaired listeners for Flanders and the Netherlands. Int J Audiol, 47, 348-355.

Vellutino, F. R., Fletcher, J. M., Snowling, M. J. et al. (2004). Specific reading disability (dyslexia): what have we learned in the past four decades? J Child Psychol Psychiatr, 45, 2 40.

Wechsler, D. (1992). Wechsler Adult Intelligence Scale (3rd Edition NL). Londen, UK: The Psychological Corporation.

Wible, B., Nicol, T., \& Kraus, N. (2004). Atypical brainstem representation of onset and formant structure of speech sounds in children with language-based learning problems. Biol Psychol, 67, 299-317.

Wible, B., Nicol, T., \& Kraus, N. (2005). Correlation between brainstem and cortical auditory processes in normal and language-impaired children. Brain, 128, 417-423.

Witton, C., Stein, J. F., Stoodley, C. J. et al. (2002). Separate influences of acoustic AM and FM sensitivity on the phonological decoding skills of impaired and normal readers. $J$ Cogn Neurosci, 14, 866-874.

Womelsdorf, T., Schoffelen, J. M., Oostenveld, R. et al. (2007). Modulation of neuronal interactions through neuronal synchronization. Science, 316, 1609-1612.

Wouters, J., Damman, W., \& Bosman, A. J. (1994). Vlaamse opname van woordenlijsten van spraakaudiometrie. Logopedie, 7, 28-33.

Ziegler, J. C. \& Goswami, U. (2005). Reading acquisition, developmental dyslexia, and skilled reading across languages: A psycholinguistic grain size theory. Psychol Bull, 131, 329. 